\title{
Bullous lungs: diverse aetiology
}

\section{S Kolekar, P Sundaram, J M Joshi}

\section{Answers on p 692.}

\section{CASE 1}

A 26 year man, a non-addict, was referred to us as he had a right sided spontaneous pneumothorax. His past medical history included surgery done at the age of 12 years for bilateral inguinal hernia and aphakia of both eyes. On physical examination there was elasticity of skin over the abdomen. Cardiac examination revealed a systolic murmur over the apex.

Routine blood tests were within normal limits. Chest radiography revealed a right sided pneumothorax, which was treated with intercostal tube drainage after which his lung expanded completely. The underlying lungs revealed the presence of bilateral bullous change (fig 1); this was confirmed on high resolution computed tomography (HRCT),

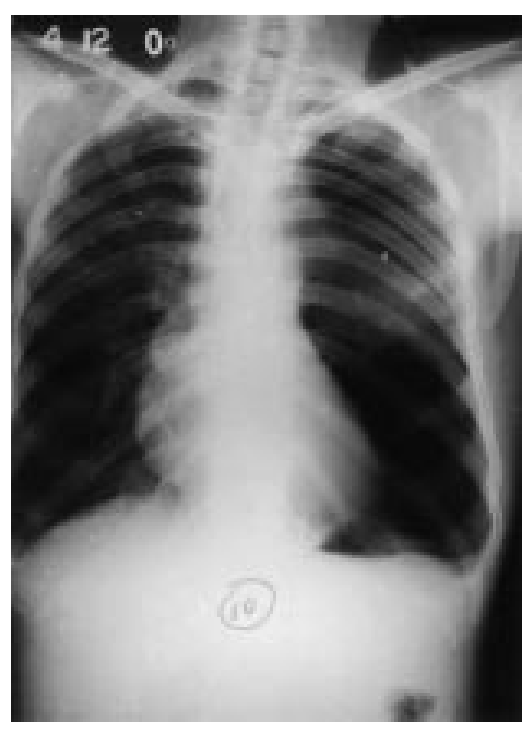

Figure 1 Chest radiograph revealing presence of bilateral bullous changes.

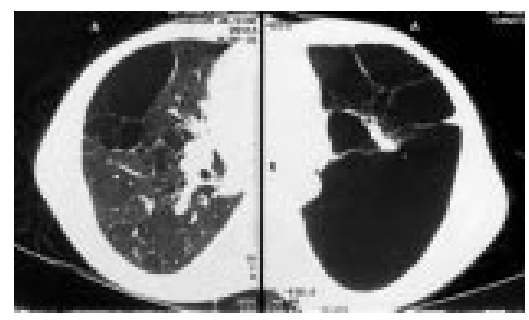

Figure 2 HRCT showing extensive bilateral bullous disease, especially marked on the left where bullae virtually replaced the lung parenchyma. which showed extensive bilateral bullous disease, especially marked in left where bullae virtually replaced the lung parenchyma (fig 2). His $\alpha_{1}$-antitrypsin levels were 199 IU/l (normal 93-224). Spirometry showed obstructive airway disease with poor bronchodilator reversibility, and increased residual volume (residual volume/total lung capacity, $92 \%$ observed) $383 \%$ predicted. Two dimensional echocardiography showed mild to moderate pulmonary hypertension and mild prolapse of mitral valve leaflets. Arterial blood gases revealed compensated type 2 respiratory failure.

\section{CASE 2}

A 34 year man, an engineer and nonsmoker, was referred to us to rule out

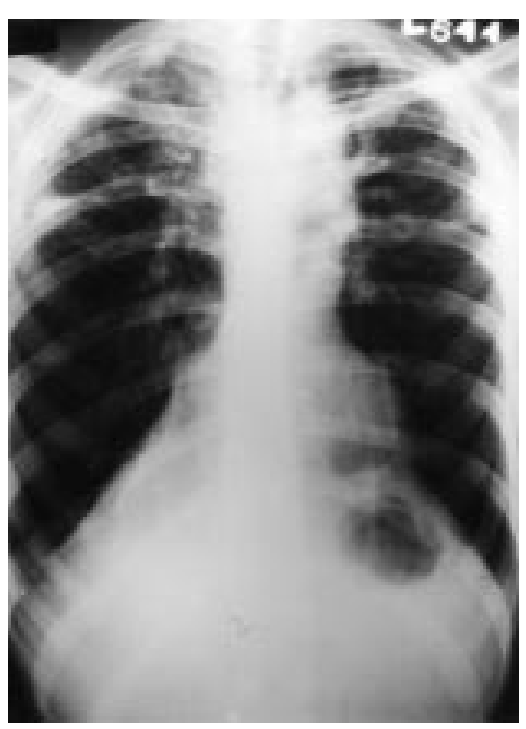

Figure 3 Chest radiography showing the presence of bilateral upper lobe fibrosis with hyperinflated lung fields.

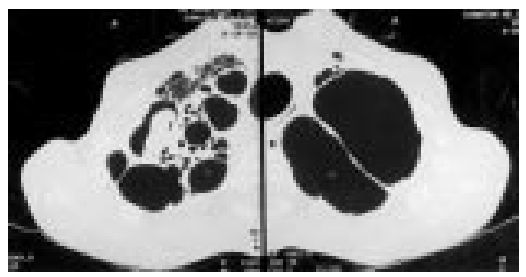

Figure 4 HRCT demonstrating the presence of fibrosis, cavities in the upper lobes, with the presence of an aspergilloma in the right upper lobe with bilateral bullae in the rest of the lung fields. drug resistant tuberculosis because of worsening of his lesions on the chest radiograph. He had symptoms of dry cough and dyspnoea for the past five years and he was treated with varying courses of antituberculosis treatment in view of the upper lobe lesions, which were progressive.

On physical examination he was a tall individual with a wide arm span and a high arched palate. There was grade 2 clubbing. On auscultation there were bilateral crepitations. On investigation baseline blood tests were within normal limits. Chest radiography revealed the presence of bilateral upper lobe fibrosis with hyperinflated lung fields (fig 3). Sputum stain and culture for acid fast bacilli were negative. HRCT demonstrated the presence of fibrosis, cavities in the upper lobes, with the presence of an aspergilloma in the right upper lobe. In addition there were bilateral bullae in the rest of the lung fields (fig 4). Two dimensional echocardiography revealed the presence of mitral regurgitation and mild pulmonary hypertension. Radiography of both hands ( fig 5) was done to calculate the metacarpal index (MCI), which was 8.6 (n-MCI $=5.4$ to 7.9$)$.

\section{QUESTION}

(1) There was extensive cystic bullous involvement of lungs in both the cases; what could be the possible diagnosis?

Postgrad Med J 2002;78:689

\section{Authors' affiliations}

S Kolekar, P Sundaram, J M Joshi, Department of Respiratory Medicine, T N Medical College and BYL Nair Hospital, Mumbai 400008, India

Correspondence to: Professor Joshi; drioshijm@email.com

Submitted 30 April 2002

Accepted 22 July 2002

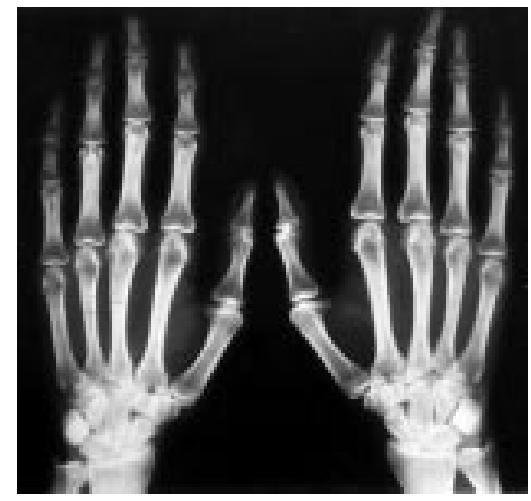

Figure 5 Radiograph of both hands. 


\section{Persistent dizziness}

Authors' affiliations

R Sivakumar, P Ghosh, S A Khan,

Department of Care of Elderly, Lister Hospital,

Stevenage, Herts SGI 4AB, UK

\section{R Sivakumar, P Ghosh, S A Khan}

Correspondence to: Dr Sivakumar; sivasiva51@hotmail.com

Submitted 4 March 2002

Answers on $p 692$.

Accepted 25 June 2002

$A$ 80 year old woman was investigated for palpitations, dizziness, and episodes of syncope. The electrocardiogram (ECG) showed atrial fibrillation. Twenty four hour Holter recording revealed atrial fibrillation throughout with intermittent pauses of more than 6 seconds. A ventricle (paced) ventricle (sensed) inhibitory and rate responsive (VVIR) pacemaker was inserted. However the patient continued to be symptomatic with episodes of dizziness and presyncope. Her ECG (see fig 1) is shown and the subsequent 24 hour tape revealed similar findings.

\section{QUESTIONS}

(1) What are the two significant electrocardiographic findings in this clinical context?

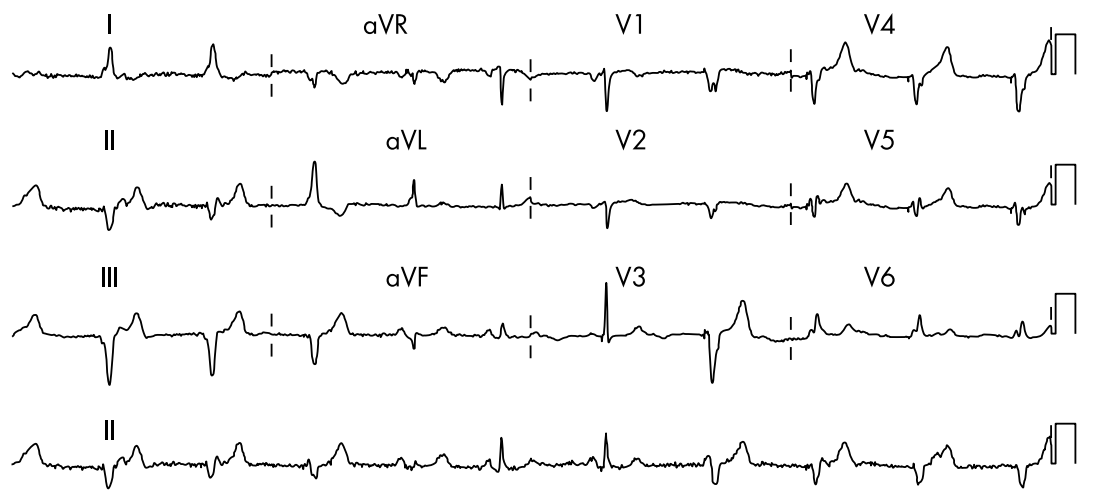

$25 \mathrm{~mm} / \mathrm{s} \quad 10 \mathrm{~mm} / \mathrm{mV}$

Figure 1 ECG of patient.

(2) What is the diagnosis?

(3) What is the definitive treatment?

Postgrad Med J 2002;78:690

\section{Young man with progressive weight loss, fevers, and a hilar mass}

\section{R T Sadikot, A P Milstone, J W Christman}

\begin{abstract}
A 27 year old white man with no significant past medical history presented with progressive malaise and fatigue for one month. Two weeks before admission he had developed a cough productive of yellow-green sputum. He had also noted fevers as high as $104.9^{\circ} \mathrm{F}\left(40.5^{\circ} \mathrm{C}\right)$ with chills and rigors. The patient reported unintentional weight loss of approximately 20 pounds $(9 \mathrm{~kg})$ over the preceding six months. He worked as a computer technician, had a 10 pack year history of smoking, with modest alcohol intake and no illicit drug use. There was no history of exposure to animals or birds.
\end{abstract}

\section{PHYSICAL EXAMINATION AND LABORATORY EVALUATION}

On admission he was febrile to $102.1^{\circ} \mathrm{F}$ $\left(38.9^{\circ} \mathrm{C}\right)$ with a pulse of 100 beats/min, blood pressure of 122/66 $\mathrm{mm} \mathrm{Hg}$, and a respiratory rate of 20 breaths $/ \mathrm{min}$. Oral examination was significant for poor dentition. Chest examination revealed dullness to percussion in the left base and crackles in the right mammary region. No clubbing or lymphadenopathy were present. The remainder of the physical examination was unremarkable. Laboratory testing revealed a white blood cell count of $16.6 \times 10^{9} /$, with $85 \%$ segmented neutrophils, lymphocytes $7 \%$, monocytes 5\%, and eosinophils $2 \%$; packed cell volume of 0.36 , and a platelet count of $437 \times 10^{9} /$. Serum chemistries and liver function tests were all normal. Serology for HIV and tuberculin skin testing were both negative. A posteroanterior and lateral chest radiograph showed a large right hilar mass and right middle lobe infiltrate with a left pleural effusion (fig l). On chest computed tomography the mass was $3 \times 3 \mathrm{~cm}$ in size with associated perihilar adenopathy. A small left sided pleural effusion was also noted (fig 2).

\section{CLINICAL COURSE}

The left sided pleural effusion was aspirated and found to be an exudate

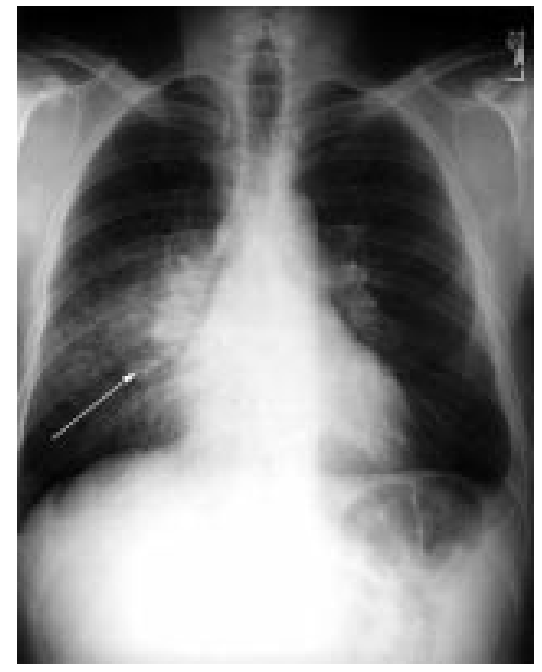

Figure 1 Chest radiograph (posteroanterior) demonstrating a right hilar mass and right middle lobe infiltrate with a left sided pleural effusion (arrow). 


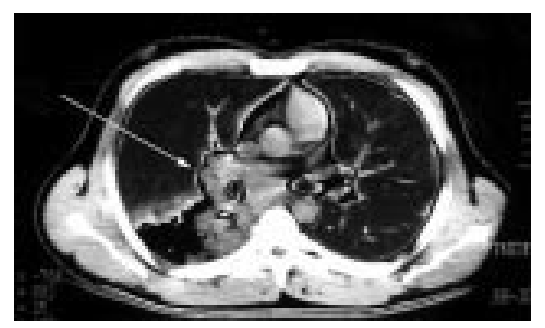

Figure 2 Computed tomogram of chest showing $2 \times 5 \mathrm{~cm}$ right hilar mass with perihilar adenopathy (arrow). Small left pleural effusion not seen on this cut.

with a protein of $43 \mathrm{~g} / \mathrm{l}$, lactate dehydrogenase of $720 \mathrm{IU} / \mathrm{l}, \mathrm{pH}$ of 8.13 , and $10.8 \mathrm{~K} / \mathrm{mm}^{3}$ white blood cells $(36 \%$ neutrophils, $46 \%$ lymphocytes, and $18 \%$ monocytes). Gram stain of the pleural fluid was negative for micro-organisms. Cultures of the pleural fluid were also negative. Fibreoptic bronchoscopy revealed a fungating mass occluding the right middle lobe bronchus. Bronchial washings and endobronchial biopsies showed Gram positive bacilli that were weakly acid fast.

\section{QUESTIONS}

(1) What is the diagnosis?

(2) What is the most appropriate therapy for this patient?

\section{ACKNOWLEDGEMENTS}

This work was supported by NIH grant T32 HL 07123 and by the Department of Veterans Affairs.

Postgrad Med J 2002;78:690-691

\section{Authors' affiliations}

R T Sadikot, J W Christman, Center for Lung Research and Division of Allergy, Pulmonary and Critical Care Medicine, Department of Medicine, Vanderbilt University Medical Center Nashville, Tennessee and Department of Veterans Affairs Medical Center, Nashville, Tennessee

A P Milstone, Center for Lung Research and Division of Allergy, Pulmonary and Critical Care Medicine, Department of Medicine, Vanderbilt University Medical Center, Nashville, Tennessee

Correspondence to: Professor John W Christman, Vanderbilt University Medical Center, Division of Allergy, Pulmonary and Critical Care, T-1217 Medical Center North Nashville, TN 37232-2650, USA;

John.Christman@mcmail.vanderbilt.edu

Submitted 17 December 2001

Accepted 13 May 2002

\section{Painful knees}

\section{S Batra, L Maini, A Upadhyay, B K Dhaon}

\section{Answers on p 694.}

$\Lambda$ 55 year old woman, suffering from osteoarthritis of the knee joints, presented with sudden worsening of pain for two months. The pain in the knee joints had gradually increased over the last 10 years. At the time of presentation the patient was severely incapacitated and could not walk without support. There was no history of trauma.
On clinical examination, there was diffuse swelling around both the knee joints. A distinct swelling was also present in the region of upper one third of the tibia on both sides. There was generalised tenderness around the knee joints. About 15 degrees of apparent varus deformity on standing and five degrees of flexion deformity on lying down was also present. Painless mobility

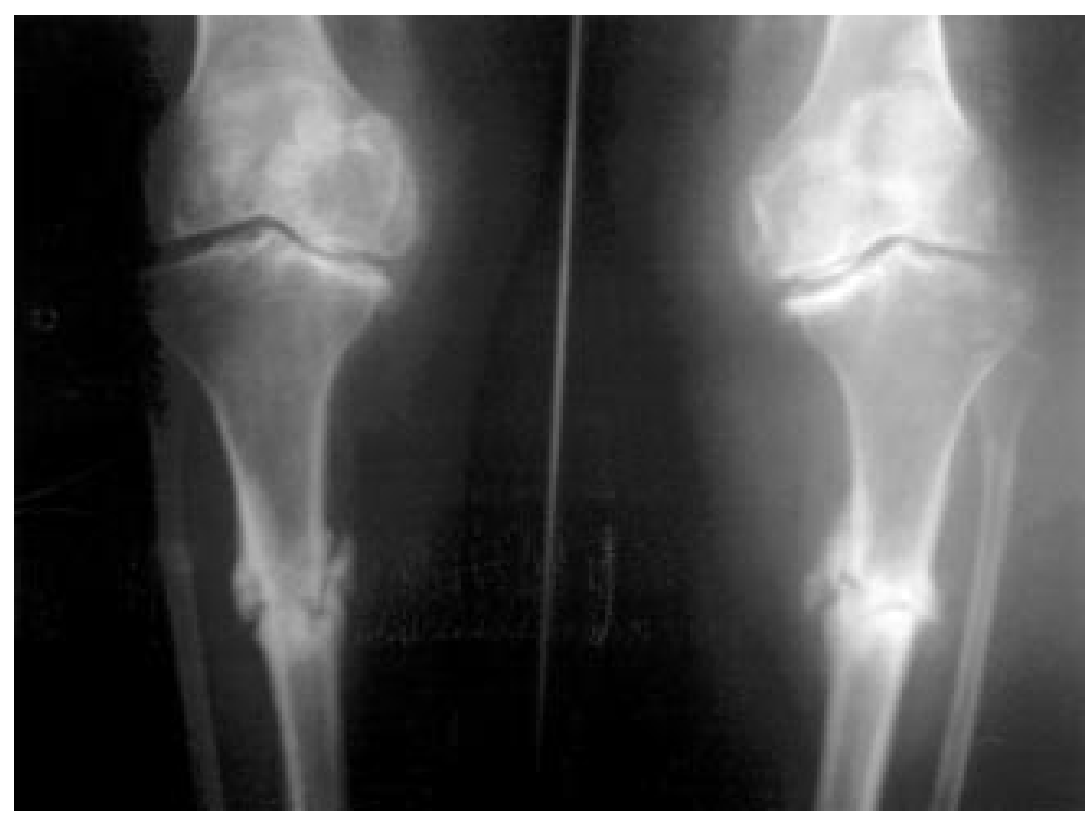

waspresent at the level of the tibial swellings. Radiographs of both lower limbs were taken and are shown in fig 1 .

\section{QUESTIONS}

(1) Describe the radiographic findings.

(2) What is the diagnosis?

(3) Discuss the pathomechanics of this condition.

(4) Discuss the treatment of this condition.

Postgrad Med J 2002;78:691

\section{Authors' affiliations}

S Batra, L Maini, A Upadhyay, B K Dhaon, Department of Orthopaedic Surgery, Maulana Azad Medical College and Associated Lok Nayak Hospital, New Delhi, India

Correspondence to: Dr Sumit Batra, B-39, Adarsh Kunj Apartments, Plot No 42, Sector 13, Rohini, Delhi 110085, India; sumitbatra104@ hotmail.com

Submitted 30 July 2001

Accepted 12 July 2002

Figure 1 Anteroposterior radiograph of both legs. 